\title{
Contribution of a Low-Barrier Hydrogen Bond to Catalysis Is Not Significant in Ketosteroid Isomerase
}

\author{
Do Soo Jang ${ }^{1,2,7}$, Gildon Choi ${ }^{3,7}$, Hyung Jin Cha ${ }^{1,4,7}$, Sejeong Shin ${ }^{5}$, Bee Hak Hong ${ }^{1,2}$, Hyeong Ju Lee ${ }^{6}$, \\ Hee Cheon Lee ${ }^{6}$, and Kwan Yong Choi ${ }^{1, *}$
}

Low-barrier hydrogen bonds (LBHBs) have been proposed to have important influences on the enormous reaction rate increases achieved by many enzymes. $\Delta^{5}$-3-ketosteroid isomerase (KSI) catalyzes the allylic isomerization of $\Delta^{5}$-3ketosteroid to its conjugated $\Delta^{4}$-isomers at a rate that approaches the diffusion limit. Tyr14, a catalytic residue of KSI, has been hypothesized to form an LBHB with the oxyanion of a dienolate steroid intermediate generated during the catalysis. The unusual chemical shift of a proton at 16.8 ppm in the nuclear magnetic resonance spectrum has been attributed to an LBHB between Tyr14 On and C3-O of equilenin, an intermediate analogue, in the active site of D38N $\mathrm{KSI}$. This shift in the spectrum was not observed in Y30F/Y55F/D38N and Y30F/Y55F/Y115F/D38N mutant KSls when each mutant was complexed with equilenin, suggesting that Tyr14 could not form LBHB with the intermediate analogue in these mutant KSIs. The crystal structure of Y30F/Y55F/Y115F/D38N-equilenin complex revealed that the distance between Tyr14 O $\eta$ and $\mathrm{C} 3-\mathrm{O}$ of the bound steroid was within a direct hydrogen bond. The conversion of LBHB to an ordinary hydrogen bond in the mutant KSI reduced the binding affinity for the steroid inhibitors by a factor of 8.1-11. In addition, the absence of LBHB reduced the catalytic activity by only a factor of 1.7-2. These results suggest that the amount of stabilization energy of the reaction intermediate provided by LBHB is small compared with that provided by an ordinary hydrogen bond in KSI.

${ }^{1}$ Department of Life Sciences, Pohang University of Science and Technology, Pohang 790-784, Korea, ${ }^{2}$ Research Institute, Genexine Co., Seongnam 463-400, Korea, ${ }^{3}$ Korea Research Institute of Chemical Technology, Daejeon 305-343, Korea, ${ }^{4}$ Pohang Accelerator Laboratory, Pohang University of Science and Technology, Pohang 790-784, Korea, ${ }^{5}$ Department of Cell Biology, Harvard Medical School, Boston, MA 02115 USA, ${ }^{6}$ Department of Chemistry, Pohang University of Science and Technology, Pohang 790-784, Korea, ${ }^{7}$ These authors contributed equally to this work.

*Correspondence: kchoi@postech.ac.kr

Received 2 October, 2014; revised 14 February, 2015; accepted 16 February, 2015; published online 7 May, 2015

Keywords: enzyme catalysis, ketosteroid isomerase, low-barrier hydrogen bond, Tyr14

\section{INTRODUCTION}

One of the fundamental questions in enzyme catalysis is how an enzyme can stabilize a reaction intermediate and transition state during enzymatic reaction to accelerate the enzyme-catalyzed reaction rate over the non-enzymatic one. The low-barrier hydrogen bond (LBHB) has been invoked to explain a large amount of the energy that is required to stabilize the intermediate in many enzyme reactions (Gerlt and Gassman, 1993). An LBHB has been hypothesized to occur when the distance between the two heteroatoms is less than the sum of their van der Waals radii and the $\mathrm{p} K_{\mathrm{a}}$ values of the hydrogen bond partners are similar to each other, so that they exhibit a covalent character that is absent in an ordinary hydrogen bond (Cleland and Kreevoy, 1994; Frey et al., 1994). The covalent character of LBHB could increase its strength; i.e., its $\Delta \mathrm{H}$ of formation increase by as much as $10-20$ $\mathrm{kcal} / \mathrm{mol}$ (Cleland et al., 1998; Zhang, 2007). Among the conventional physicochemical parameters used to characterize LBHBs, the unusual chemical shift of proton nuclear magnetic resonance (NMR) near 16 to $20 \mathrm{ppm}$ in the downfield region is regarded as the clearest evidence for the presence of LBHBs in proteins (Cleland and Kreevoy, 1994; Frey et al., 1994). However, the degree to which LBHB contributes to catalysis is controversial (Ash et al., 1997; Perrin, 2010; Pollack et al., 1999; Schutz and Warshel, 2004). A theoretical calculation led to a conclusion that LBHB cannot stabilize the transition-state more than can ordinary hydrogen bonds (Warshel and Papazyan, 1996). Experimental evidence in model compounds suggested that LBHBs might not contribute additional energy to the hydrogen bond between a hydrogen bond donor and acceptor that have matched $\mathrm{p} K_{a} \mathrm{~s}$ (Shan et al., 1996).

$\Delta^{5}$-3-ketosteroid isomerase (KSI) catalyzes the allylic rearrangement of the 5,6 double bond of $\Delta^{5}$-3-ketosteroids to the 4,5 position, and accelerates the reaction by a factor of $10^{11}$ compared to the nonenzymatic reaction by an intramolecular proton transfer (Fig. 1) (Pollack, 2004). Two bacterial KSIs, one from Comamonas testosteroni and one from Pseudomonas putida have been studied to understand the enzyme-catalyzed heterolytic $\mathrm{C}-\mathrm{H}$ bond cleavage that occurs in a wide variety of biological reactions (Gerlt et al., 1991). In the reaction catalyzed by KSI, Tyr14 and Asp99 are thought to have critical functions in stabilizing a dienolate intermediate by forming LBHB or ordinary hydrogen bonds with the oxyanion of the intermediate (Cho et al., 1998; Kim et al., 1997a). The ${ }^{1}$ H NMR spectrum of 


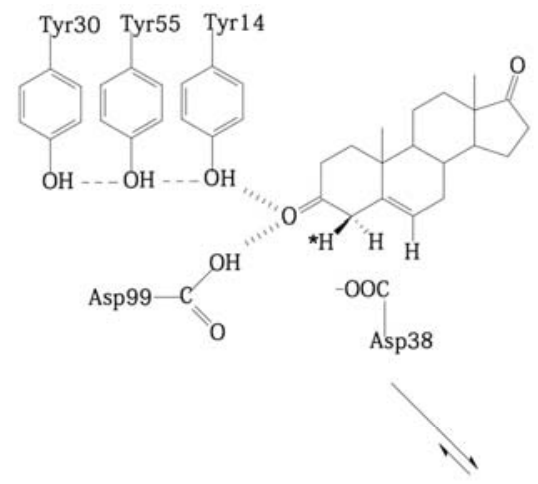

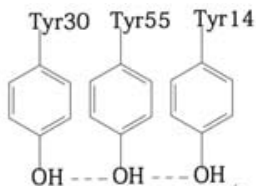<smiles>C1CCCCC1</smiles>

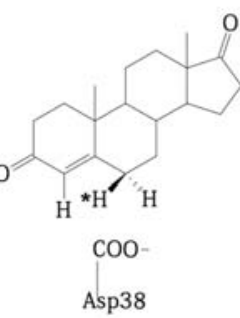<smiles>C=CC</smiles>

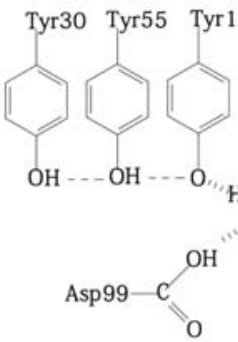<smiles>CC12CCC(=O)C=C1C=CC1C3CCC(=O)CC3C1(C)CCC2=O</smiles>

Fig. 1. Reaction catalyzed by ketosteroid isomerase. Androste- nolone, equilenin, and estrone are analogues of substrate, intermediate, and product of $\mathrm{KSI}$, respectively. The $\beta$ proton at $\mathrm{C}-4$ is transferred by Asp 38 to the $\beta$ side of $C-6$ during the isomerization reaction. Both Tyr14 and Asp99 can stabilize the intermediate by forming a hydrogen bond with the oxyanion of the intermediate. Tyr14 is hydrogen-bonded to Tyr55 that is in turn hydrogen bonded to Tyr30 in the P. putida KSI.
KSI complexed with equilenin (i.e., an intermediate analogue in the reaction) shows a highly deshielded proton resonance near $17 \mathrm{ppm}$, which has been regarded as compelling evidence for the involvement of LBHB in the catalysis (Cho et al., 1999; Zhao et al., 1996; 1997). NMR spectroscopic studies combined with site-directed mutagenesis have revealed that an LBHB can form between Tyr14 On and C3-O of equilenin in the active site of D38N (Ha et al., 2001). The strength of the LBHB in C. testosteroni $\mathrm{KSI}$ has been estimated to be at least $7.1 \mathrm{kcal} / \mathrm{mol}$ by comparing the dissociation rates of the intermediate from the Y14F and the D38N mutants (Xue et al., 1991) and by measuring the proton exchange rate of the LBHB over the $\mathrm{pH}$ range 4.3 to 9.0 (Zhao et al., 1996; 1997). The Y14F mutation reduced $k_{\text {cat }}$ of $C$. testosteroni KSI by a factor of $5 \times 10^{4}$ (Kuliopulos et al., 1989) but that of the D99A mutation only a factor of $5 \times 10^{3}$ (Wu et al., 1997). In addition, Y14F and D99A mutants of $P$. putida KSI (in this paper we number the residues of $P$. putida $\mathrm{KSI}$ according to those of $C$. testosteroni $\mathrm{KSI}$ ) are only $1 / 2,000$ and $1 / 98$ times as active as the wild-type KSI (Kim and Choi, 1995; Kim et al., 1997b), respectively; this change suggests that by forming LBHBs, Tyr14 contributes to catalysis more crucially than does Asp99.

In this study, we measured the energetic difference between LBHB and the ordinary hydrogen bond in the active site of $P$. putida KSI. Together with the structural analyses for the hydrogen bonds involved in the catalytic reaction of $P$. putida KSI, our NMR spectroscopic studies revealed that the putative LBHB between Tyr14 O $\eta$ and C3-O of equilenin observed for D38N KSI was converted to an ordinary hydrogen bond by the Y30F/Y55F mutations. The conversion of the LBHB to an ordinary hydrogen bond resulted in only marginal effects on both catalytic activity of KSI and its binding affinity for the intermediate analogue. Our results suggest that the contribution of LBHB to catalysis should be only marginal compared with that of an ordinary hydrogen bond in the active site of $\mathrm{KSI}$.

\section{MATERIALS AND METHODS}

\section{Materials}

5-androstene-3,17-dione (5-AND), androstenolone, equilenin and estrone were purchased from Steraloids Inc. (USA). ${ }^{15} \mathrm{~N}$-Labeled $\mathrm{NH}_{4} \mathrm{Cl}$ was purchased from Cambridge Isotope Laboratories Inc. (USA). A Superose 12 gel filtration column was purchased from Amersham Bioscience (USA). All chemicals for the buffer solution were purchased from Sigma (USA). All enzymes for DNA manipulation were purchased from Promega (USA). Oligonucleotides were obtained from Genotech Inc. (Korea).

Site-directed mutagenesis, expression and purification of mutant KSIs

Site-directed mutagenesis of $\mathrm{Y} 115 \mathrm{~F}, \mathrm{Y} 115 \mathrm{~F} / \mathrm{D} 38 \mathrm{~N}, \mathrm{Y30F} / \mathrm{Y} 55 \mathrm{~F} /$ Y115F, Y30F/Y55F/D38N and Y30F/Y55F/Y115F/D38N was conducted as described previously (Kim et al., 2000). All mutations were confirmed by sequencing the entire gene of the mutant KSI. Mutant KSls were overexpressed in Escherichia coli $\mathrm{BL21(DE3)} \mathrm{(Novagen)} \mathrm{harboring} \mathrm{an} \mathrm{expression} \mathrm{vector} \mathrm{plasmid}$ containing the mutant KSI gene, and were purified as described previously (Cha et al., 2013; Jang et al., 2004). The purity of KSI was confirmed by the presence of only one band in SDS-PAGE.

NMR spectroscopy

NMR was performed as described previously (Jang et al., 2006). The NMR sample was adjusted to contain $15 \mathrm{mg} / \mathrm{ml} \mathrm{KSI}$ in $40 \mathrm{mM}$ potassium phosphate buffer at $\mathrm{pH} 7.0$ containing 1 $\mathrm{mM}$ EDTA and 10\% DMSO-d $\mathrm{d}_{6}$ (dimethyl sulfoxide- $\left.\mathrm{d}_{6}\right)(\mathrm{v} / \mathrm{v})$. NMR spectra were collected on a spectrometer (Bruker, Avance DRX500) equipped with a triple resonance, pulse field gradient probe with actively-shielded z-axis gradients, and a gradient amplifier unit. A jump-and-return pulse sequence (Plateau and Gueron, 1982) was used to suppress the water signal. The observed ${ }^{1} \mathrm{H}$ chemical shifts were determined relative to that of sodium 2,2-dimethyl-2-silapentane-5-sulfonate as an 
internal standard. All experiments were conducted at $270 \mathrm{~K}$ with 1,000 scans acquired for each spectrum; the relaxation delay was $2 \mathrm{~s}$. The spectral width of $20,000 \mathrm{~Hz}$ was used in 16,384 points. Line-broadening of $10 \mathrm{~Hz}$ was used when processing the data on a workstation (Silicon Graphics, IndyPC) using a software program XWIN-NMR v. 1.2.

Determination of dissociation constants for steroid inhibitors The affinity of KSI for equilenin, androstenolone, or estrone was assessed by measuring the extent of quenching of intrinsic tryptophan fluorescence of KSI upon the binding of the respective steroid. The fluorescence intensities of KSI were measured with excitation at $295 \mathrm{~nm}$ and emission at $315 \mathrm{~nm}$ after adding successively various amounts of the steroid dissolved in DMSO to the solution of KSI. After correcting the data with appropriate dilution factors, dissociation constants were determined by fitting the data to the following equation:

$$
[1]_{\mathrm{t}}=\left(F_{0}-F\right)\left\{K_{\mathrm{D}} /\left(F-F_{\infty}\right)+[E] /\left(F_{0}-F_{\infty}\right)\right\},
$$

where []$_{t}$ is the concentration of total steroid in the solution, $F$ is the fluorescence intensity, $F_{0}$ is the intensity in the absence of any steroid, $F_{\infty}$ is the intensity in infinite steroid concentration, $[E]$ is the concentration of $\mathrm{KSI}$, and $K_{\mathrm{D}}$ is the dissociation constant.

Determination of kinetic parameters, $\boldsymbol{k}_{\mathrm{cat}}$ and $\boldsymbol{K}_{\mathrm{M}}$ Enzymatic reactions were initiated by adding $\mathrm{KSI}$ to $3 \mathrm{ml}$ of a solution containing $34 \mathrm{mM}$ potassium phosphate, $\mathrm{pH} 7.0,2.5$ mM EDTA, 3.3\% methanol, and the respective amount of a steroid substrate, 5-AND. The final concentration of methanol in the reaction mixture was $3.3 \%$ by volume. All the assays were performed at $298 \mathrm{~K}$. Kinetic parameters such as $k_{\text {cat }}$ and $K_{M}$ were obtained by utilizing Lineweaver-Burk reciprocal plots under the assay conditions in which the substrate concentrations were $23.3,34.9,58.2,81.5$, and $116.4 \mu \mathrm{M}$.

Crystallization and structure determination of mutant KSIs Crystallization of Y30F/Y55F/Y115F/D38N KSI complexed with equilenin was conducted using a hanging drop vapor diffusion method as described previously (Cha et al., 2014; Cho et al., 1999). After $20 \mathrm{mg} / \mathrm{ml}$ of the mutant $\mathrm{KSI}$ was prepared in a buffer containing $40 \mathrm{mM}$ potassium phosphate, $\mathrm{pH} 7.0,1 \mathrm{mM}$ EDTA, and $20 \mathrm{mM} \beta$-mercaptoethanol, the $70 \mu \mathrm{l}$ of this solution was mixed with $2 \mu \mathrm{l}$ of $10 \mathrm{mM}$ equilenin in dimethyl sulfoxide. The optimized crystallization for the mutant $\mathrm{KSI}$ was $0.1 \mathrm{M}$ sodium acetate, $\mathrm{pH}$ 4.5, 0.6 M ammonium acetate, and 30\% PEG 4000. All diffraction data were collected on beamline $5 \mathrm{C}$ at Pohang Accelerator Laboratory (PAL), Korea. The diffraction images were integrated and scaled using the HKL-2000 software package (Otwinowski and Minor, 1997). The structure of Y30F/Y55F/Y115F/D38N complexed with equilenin was determined by the molecular replacement method using as a search model the coordinates of wild-type KSI that had been determined previously (Kim et al., 1997a). Model building was performed using Coot (Emsley et al., 2010) and refinement was performed using Phenix.refine (Afonine et al., 2012). The atomic coordinate of Y30F/Y55F/Y115F/D38N complexed with equilenin was deposited at the Protein Data Bank (PDB code 5AI1).

\section{RESULTS}

Effects of tyrosine-to-phenylalanine substitution(s) in $P$. putida KSI on LBHB

The Y30F/Y55F/Y115F/D38N mutant KSI that contains no

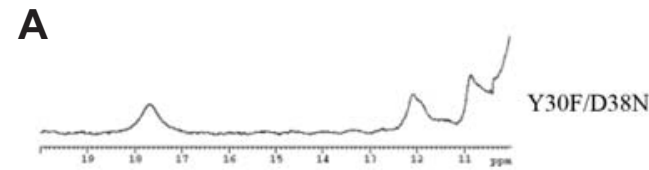

B
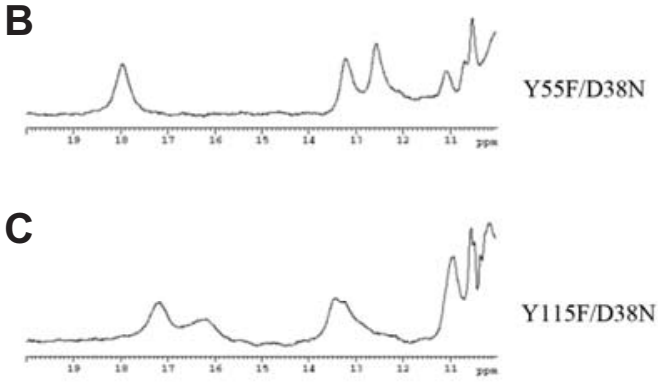

D

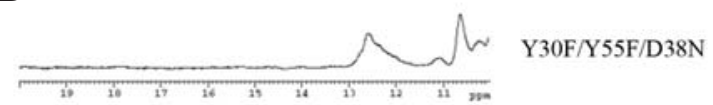

$\mathbf{E}$

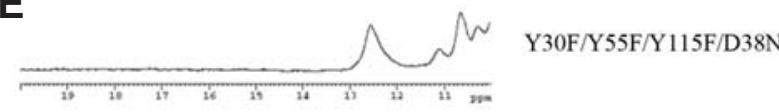

Fig. 2. ${ }^{1} \mathrm{H}$ NMR spectra of $P$. putida KSIs. (A) Y30F/D38N at pH 8.5, (B) $Y 55 F / D 38 \mathrm{~N}$ at $\mathrm{pH} 7.0$, (C) $Y 115 F / D 38 \mathrm{~N}$ at $\mathrm{pH} 8.5$, (D) Y30F/Y55F/D38N at pH 8.5, (E) Y30F/Y55F/Y115F/D38N at pH 7.0, which are complexed with equilenin, respectively. The concentration of $\mathrm{KSI}$ is $10 \mathrm{mg} / \mathrm{ml}$ and the concentration of DMSO- $\mathrm{d}_{6} 10 \%$.

tyrosine residue other than Tyr14 was prepared to investigate the role of Tyr14 in the formation of LBHB without any interference from the other three tyrosines (Tyr30, Tyr55, Tyr115) in $P$. putida KSI. A highly deshielded proton resonance was not observed in the downfield region of the NMR spectrum of Y30F/Y55F/Y115F/D38N KSI complexed with equilenin (Fig. 2E), although Tyr14 still has a hydrogen bonding capability in the active site of the mutant. To determine why LBHB is absent in the Y30F/Y55F/Y115F/D38N-equilenin complex, the tyrosines at positions 30,55 , and 115 in D38N were replaced individually with a phenylalanine. The ${ }^{1} \mathrm{H}$ NMR spectra of the Y30F/D38N, Y55F/D38N, and Y115F/D38N mutants revealed that LBHB between Tyr14 On and C3-O of equilenin was not perturbed significantly by the Y30F, Y55F, or Y115F mutation (Figs. 2A, 2B, and $2 \mathrm{C})$. In contrast to the single substitutions of tyrosine with phenylalanine, the highly deshielded proton resonance was not observed in the ${ }^{1} \mathrm{H}$ NMR spectrum of Y30F/Y55F/D38N-equilenin complex (Fig. 2D); this result indicates that the absence of LBHB in Y30F/Y55F/Y115F/D38N can be attributed to the simultaneous replacements of tyrosines with phenylalanines at the positions 30 and 55 in P. putida KSI.

Affinity of steroid to KSI

$K_{\mathrm{D}}$ values of $\mathrm{KSI}$ for equilenin and other steroid inhibitors were determined by analyzing the quenching of the intrinsic fluores- 
Contribution of LBHB to Catalysis Is Not Significant in KSI

Do Soo Jang et al.

Table 1. Effects of the tyrosine-to-phenylalanine substitutions in P. putida KSI on the binding of equilenin and the low-barrier hydrogen bond ${ }^{a}$

\begin{tabular}{|c|c|c|}
\hline KSI & $\begin{array}{c}K_{\mathrm{D}}^{\mathrm{b}} \\
(\mu \mathrm{M}) \\
\end{array}$ & $\begin{array}{l}\text { Highly deshielded } \\
{ }^{1} \mathrm{H} \text { resonance }(\mathrm{ppm})^{\mathrm{C}}\end{array}$ \\
\hline D38N & $(3.1 \pm 0.6) \times 10^{-2}$ & 16.8 \\
\hline Y30F/D38N & $(0.7 \pm 0.1) \times 10^{-2}$ & 17.6 \\
\hline Y55F/D38N & $(0.8 \pm 0.2) \times 10^{-2}$ & 17.9 \\
\hline Y115F/D38N & $(0.7 \pm 0.2) \times 10^{-2}$ & $17.1,16.2$ \\
\hline Y30F/Y55F/D38N & $(2.5 \pm 0.7) \times 10^{-1}$ & Not Detected \\
\hline Y30F/Y55F/Y115F/D38N & $(3.4 \pm 0.7) \times 10^{-1}$ & Not Detected \\
\hline
\end{tabular}

\footnotetext{
${ }^{\mathrm{a}}$ The $K_{\mathrm{D}}$ measurements and NMR experiments were performed in a buffer containing $40 \mathrm{mM}$ potassium phosphate, $1 \mathrm{mM}$ EDTA, and $10 \%$ DMSO.

${ }^{b}$ Values are mean \pm standard deviation from three independent experiments.

${ }^{\mathrm{c}}$ Only the ${ }^{1} \mathrm{H}$ NMR resonance ranging from 16 to $20 \mathrm{ppm}$, which is regarded as the most unambiguous evidence for the presence of LBHBs in proteins, were investigated to identify the presence of LBHB in the mutant KSIs.
}

Table 2. Affinities of D38N and Y30F/Y55F/Y115F/D38N KSI for the steroid inhibitors ${ }^{\mathrm{a}}$

\begin{tabular}{lccc}
\hline \multicolumn{1}{c}{ Enzyme } & Steroid & $K_{\mathrm{D}}(\mu \mathrm{M})^{\mathrm{b}}$ & $\begin{array}{c}\text { Highly deshielded } \\
{ }^{1} \mathrm{H} \text { resonance }(\mathrm{ppm})^{\mathrm{c}}\end{array}$ \\
\hline \multirow{2}{*}{ D38N } & equilenin & $(3.1 \pm 0.6) \times 10^{-2}$ & 16.8 \\
& androstenolone & $(3.0 \pm 0.7) \times 10^{-1}$ & 16.8 \\
Y30F/Y55F/Y115F/D38N & estrone & $(1.1 \pm 0.4) \times 10^{-1}$ & 18.0 \\
& equilenin & $(3.4 \pm 0.7) \times 10^{-1}$ & Not Detected \\
& androstenolone & $3.2 \pm 0.3$ & Not Detected \\
\end{tabular}

\footnotetext{
${ }^{a}$ Measurements were performed in $10 \mathrm{mM}$ potassium phosphate buffer containing $10 \%$ dimethyl sulfoxide.

${ }^{b}$ Values are mean \pm standard deviation from three independent experiments.

${ }^{\mathrm{c}}$ Only the ${ }^{1} \mathrm{H}$ NMR resonances ranging from 16 to $20 \mathrm{ppm}$ were investigated to identify the presence of LBHB in KSI.
}

cence of the enzyme while varying the steroid concentration. The conditions such as the concentration of DMSO in the fluorescence measurements were the same as those in the NMR experiments. When the mutation of Y30F, Y55F or Y115F was imposed in addition to $\mathrm{D} 38 \mathrm{~N}$, the $K_{\mathrm{D}}$ values of the mutant $\mathrm{KSIs}$ were only approximately doubled relative to that of D38N (Table 1). The $K_{\mathrm{D}}$ values of the $\mathrm{Y} 30 \mathrm{~F} / \mathrm{Y} 55 \mathrm{~F} / \mathrm{D} 38 \mathrm{~N}$ and $\mathrm{Y} 30 \mathrm{~F} /$ Y55F/Y115F/D38N mutants whose ${ }^{1} \mathrm{H}$ NMR spectra exhibited no highly deshielded proton resonance in the downfield region around $16 \mathrm{ppm}$, were increased by factors of 8.1 and 11 , respectively (Table 1).

The $K_{\mathrm{D}}$ values of D38N and Y30F/Y55F/Y115F/D38N were also determined for the steroid inhibitors androstenolone and estrone (Fig. 3). When androstenolone or estrone was bound to $\mathrm{D} 38 \mathrm{~N} \mathrm{KSI}$, a highly deshielded proton resonance at 16.8 or $18.0 \mathrm{ppm}$ respectively, was observed in the downfield of the ${ }^{1} \mathrm{H}$ NMR spectrum. In contrast, the highly deshielded proton resonance was not observed in the ${ }^{1} \mathrm{H}$ NMR spectrum of Y30F/Y55F/Y115F/D38N complexed with androstenolone or estrone, respectively. Even if the $A$ and $B$ ring structures of androstenolone and estrone are very different from those of equilenin, the additional tyrosine-to-phenylalanine substitutions reduced the binding affinities of D38N KSI for these steroid inhibitors to a similar extent as that for equilenin (Table 2).

\section{Kinetic analyses}

The $k_{\text {cat }}$ and $K_{\mathrm{M}}$ values of the mutant KSls for a steroid substrate (5-AND) were compared with those of the wild type (Table 3). A previous investigation on the hydrogen bond network (Kim et al., 2000) revealed that the $k_{\text {cat }}$ values of Y30F and Y55F were decreased by factors of 1.2 and 6 respectively rela-

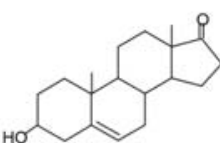

androstenolone

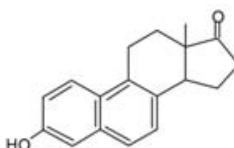

$d$-equilenin

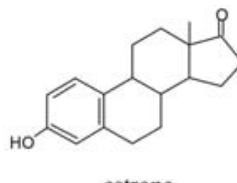

estrone
Fig. 3. Structures of important steroid inhibitors of KSI. All the steroid inhibitors have the hydroxyl group at the C3 position that can form a hydrogen bond with Tyr14 and Asp99. The binding affinities of the steroid inhibitors for D38N were lowered to the similar extent by the additional $\mathrm{Y} 30 \mathrm{~F} / \mathrm{Y} 55 \mathrm{~F} / \mathrm{Y} 115 \mathrm{~F}$ mutations irrespective of the structural differences among the inhibitors.

tive to that of the wild-type KSI. These $k_{\text {cat }}$ values are signifycantly higher than that of $\mathrm{Y} 14 \mathrm{~F}$ which is lower by a factor of $10^{3.2}$ than that of the wild-type $\mathrm{KSI}$. In addition, the replacement of Tyr115 with a phenylalanine also resulted in decrease in $k_{\text {cat }}$ by a factor of 1.5 . These results indicate that Tyr30, Tyr55 and Tyr115 are not critically essential for catalysis by $P$. putida KSI even if the contribution of Tyr55 to catalysis is somewhat significant. In spite of the absence of LBHB in the ${ }^{1} \mathrm{H}$ NMR spectra, Y30F/Y55F and Y30F/Y55F/Y115F mutant KSIs exhibited relatively high catalytic activities with the $k_{\text {cat }}$ values decreased marginally by factors of 2.0 and 1.7, respectively, compared with the wild type. These $k_{\text {cat }}$ values were even significantly higher than that of $\mathrm{Y} 55 \mathrm{~F}$ whose ${ }^{1} \mathrm{H}$ NMR spectrum exhibited a highly deshielded proton resonance at $17.9 \mathrm{ppm}$, which is similar to that of the wild-type enzyme. 
Table 3. Kinetic parameters of the wild type and its mutant $\mathrm{KSIs}^{\mathrm{a}}$

\begin{tabular}{lcccc}
\hline \multicolumn{1}{c}{ Enzyme } & $\begin{array}{c}k_{\text {cat }} \\
\left(\mathrm{s}^{-1}\right)\end{array}$ & $\begin{array}{c}K_{\mathrm{M}} \\
(\mu \mathrm{M})\end{array}$ & $\begin{array}{c}k_{\text {cat }} / K_{\mathrm{M}} \\
\left(\mathrm{M}^{-1} \mathrm{~s}^{-1}\right)\end{array}$ & $\begin{array}{c}\text { Relative } \\
k_{\text {cat }}\end{array}$ \\
\hline WT $^{\mathrm{b}}$ & $(21.2 \pm 0.8) \times 10^{3}$ & $49.9 \pm 1.3$ & $4.3 \times 10^{8}$ & 1 \\
Y14F $^{\mathrm{c}}$ & $13.3 \pm 0.6$ & $17.1 \pm 3.1$ & $7.8 \times 10^{5}$ & $10^{-3.2}$ \\
Y30F $^{\mathrm{d}}$ & $(17.8 \pm 0.1) \times 10^{3}$ & $55.2 \pm 2.0$ & $3.3 \times 10^{8}$ & 0.84 \\
Y55F $^{d}$ & $(35.1 \pm 0.6) \times 10^{2}$ & $23.0 \pm 1.0$ & $1.5 \times 10^{8}$ & 0.17 \\
Y115F & $(14.5 \pm 0.4) \times 10^{3}$ & $41.7 \pm 2.0$ & $3.5 \times 10^{8}$ & 0.68 \\
Y30F/Y55F & $(10.7 \pm 0.4) \times 10^{3}$ & $50.2 \pm 5.5$ & $2.1 \times 10^{8}$ & 0.50 \\
Y30F/Y55F/Y115F & $(12.2 \pm 0.2) \times 10^{3}$ & $77.3 \pm 5.6$ & $1.6 \times 10^{8}$ & 0.57 \\
\hline
\end{tabular}

${ }^{a}$ The assays were performed in a buffer containing $34 \mathrm{mM}$ potassium phosphate, pH 7.0, $2.5 \mathrm{mM}$ EDTA and $3.3 \%$ methanol. ${ }^{\mathrm{b}}$ Data from Kim et al. (1999).

${ }^{\mathrm{c}}$ Data from Kim and Choi (1995). ${ }^{\mathrm{d}}$ Data from Kim et al. (2000).
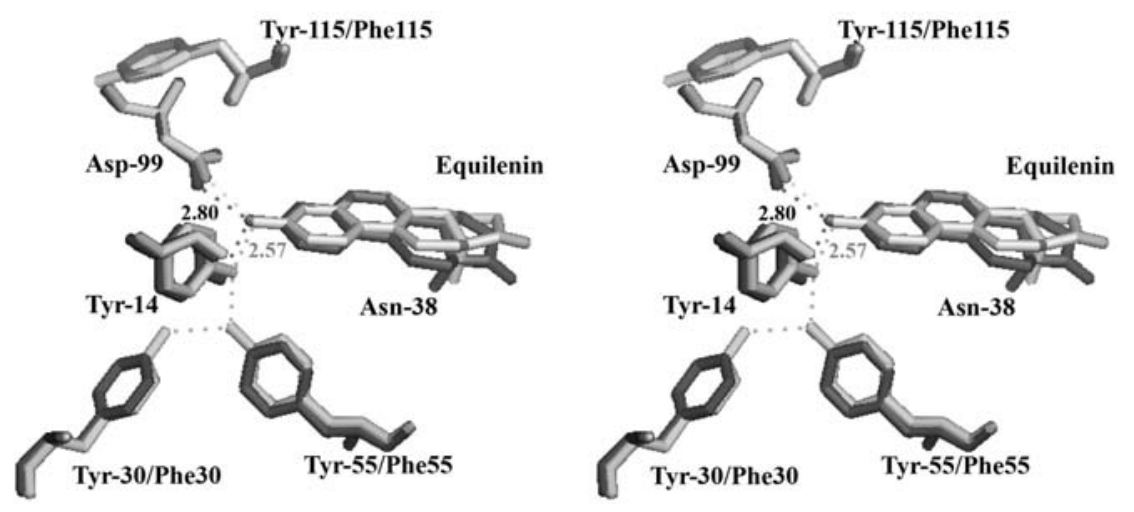

Fig. 4. Crystal Structure of $\mathrm{Y} 30 \mathrm{~F} / \mathrm{Y} 55 \mathrm{~F} /$ Y115F/D38N complexed with equilenin. All the backbone atoms were utilized to superimpose the structures of D38N (PDB code 1E3N) and Y30F/Y55F/Y115F/ D38N, which are complexed with equilenin. The active-site residues of $\mathrm{D} 38 \mathrm{~N}$ are colored in gray and those of Y30F/Y55F/Y115F/D38N in black. The hydrogen bonds are indicated by dotted lines. The PyMOL program was used to display the structures.
Three-dimensional structure of Y30F/Y55F/Y115F/D38N complexed with equilenin

The binding mode of equilenin in the active site of Y30F/ Y55F/Y115F/D38N was investigated using $X$-ray crystallography. The crystals of Y30F/Y55F/Y115F/D38N-equilenin complex were found to belong to the $C 222_{1}$ space group with unit cell dimensions of $a=35.2 \AA, b=95.8 \AA$ and $c=73.5 \AA$. The final structure of $Y 30 F / Y 55 F / Y 115 F / D 38 N-e q u i l e n i n$ complex was determined at 2.1-Å resolution. The crystallographic statistics for the final structure are shown in Supplementary Table 1. The crystal structure of the mutant revealed that the benzyl side chains of the substituted phenylalanines were located at the positions similar to those of the corresponding tyrosine residues in D38N but without the hydroxyl groups (Fig. 4). The hydrogen bond geometry between equilenin and catalytic residues in Y30F/Y55F/Y115F/D38N was very similar to that in D38N. The bound steroid was located in the active site of the mutant with the steroid A-ring approaching Tyr14 and Asp99 (Fig. 4; Supplementary Fig. 1). Three catalytic residues, Tyr14, Asp38 and Asp99, which are critical for the catalytic activity of KSI, were found to be located at positions similar to those in D38N. Tyr14 On was involved in a hydrogen bond with $\mathrm{C} 3-\mathrm{O}$ of equilenin with a distance of $2.80 \AA$ in the active site of $\mathrm{Y} 30 \mathrm{~F} / \mathrm{Y} 55 \mathrm{~F} /$ Y115F/D38N complexed with the steroid.

\section{DISCUSSION}

Using $\mathrm{KSI}$ as a model system, we investigated the nature and origin of stabilization of the reaction intermediate or the transition-state to explain the enormous rate enhancement of the enzyme-catalyzed reaction over the non-enzymatic reaction. Especially, we investigated the proposition that LBHB can provide an additional energetic contribution to enzyme catalysis compared with the ordinary hydrogen bond. In P. putida KSI, LBHB was proposed to be formed between Tyr14 On and C3$\mathrm{O}$ of equilenin, an intermediate analogue, in the active site of D38N KSI, as revealed by NMR spectroscopic studies combined with site-directed mutagenesis (Ha et al., 2001). The absence of a highly deshieled proton resonance at $\sim 16 \mathrm{ppm}$ in the ${ }^{1} \mathrm{H}$ NMR spectrum of Y30F/Y55F/Y115F/D38N KSI complexed with equilenin clearly indicates that the LBHB between Tyr14 On and C3-O of the steroid does not exist in the active site of the mutant $\mathrm{KSI}$. In addition, the distance between Tyr14 O $\eta$ and $\mathrm{C} 3-\mathrm{O}$ of equilenin was changed. The distance in the Y30F/ Y55F/Y115F/D38N KSI was $2.80 \AA$, whereas that in D38N KSI was $2.57 \AA$ (Fig. 4). Considering that the major features of a LBHB are a distance $<2.65 \AA$ between a proton donor and a proton acceptor or a 16- to 20-ppm ${ }^{1} \mathrm{H}$ NMR chemical shift in the downfield region, or both (Frey et al., 1994; Fuhrmann et al., 2006), the putative LBHB might be replaced by an ordinary hydrogen bond in Y30F/Y55F/Y115F/D38N KSI.

Tyr14 On forms a hydrogen bond with Tyr55 On that is in turn hydrogen-bonded to Tyr30 O $\eta$ in the active site of $P$. putida $\mathrm{KSI}$ (Fig. 1). Tyr55 may have an important function in the formation of LBHB between Tyr14 On and C3-O of equilenin by providing a hydrogen bond to Tyr14 and consequently affecting the 
$\mathrm{pK} K_{\mathrm{a}}$ of Tyr14 through the hydrogen bond. The crystal structure of Y55F P. putida KSI (Kim et al., 2000) revealed a new hydrogen bond between Tyr14 On and Tyr30 On; this bond compensates for the loss of a hydrogen bond that was provided by Tyr55. Simultaneous replacements of Tyr30 and Tyr55 with phenylalanines in $P$. putida $\mathrm{KSI}$ can both disrupt the hydrogen bond between Tyr14 and Tyr55 and change the local dielectric constant of the catalytic site of the mutant KSI; both of these changes can influence the $p K_{a} s$ of the hydrogen bond partners of the LBHB. Our data suggest that the hydrogen bond between Tyr14 and Tyr55 could be essential to enable the formation of LBHB in the active site of KSI.

The ${ }^{1} \mathrm{H}$ NMR spectrum and crystal structure of the Y30F/Y55F/Y115F/D38N KSI complexed with equilenin revealed that $\mathrm{LBHB}$ between Tyr14 On and C3-O of the steroid in D38N was replaced by an ordinary hydrogen bond. The affinities of the Y30F/Y55F/Y115F/D38N KSI for steroid inhibitors (androstenolone, estrone, equilenin), were lower by factors of 9.1-11 than those of the $\mathrm{D} 38 \mathrm{~N} \mathrm{KSI}$; this change corresponds to a decrease of only 1.3-1.4 kcal/mol in binding energy. This decrease in the binding energy could be attributed to the additional contribution of the LBHB to the steroid binding compared with that of the ordinary hydrogen bond in the active site of KSI. In addition, the catalytic activities of the Y30F/Y55F and Y30F/Y55F/Y115F KSIs were only slightly decreased compared with that of the wild-type enzyme in spite of the conversion of LBHB to an ordinary hydrogen bond in these mutant KSIs. Similar observations have also been reported in other enzyme systems (Stratton et al., 2001; Zhao et al., 2004). A serine protease, subtilisin BPN' demonstrated a downfield NMR resonance near $18 \mathrm{ppm}$ that was considered to originate from LBHB in the wild-type subtilisin (Stratton et al., 2001). This characteristic downfield chemical shift is not present in the D32C subtilisin, but this mutation had only moderate effects on the catalytic activity of subtilisin; this observation suggests that an LBHB does not provide exceptionally high stabilization energy compared with an ordinary hydrogen bond (Stratton et al. 2001). Carboxymethyldethia coenzyme $A$, an inhibitor of citrate synthase, forms an LBHB with Asp375 of the enzyme, whereas the primary amine analog of the coenzyme A forms an ordinary hydrogen bond (Usher et al., 1994). Only the extra energy of $1.8 \mathrm{kcal} / \mathrm{mol}$ has been attributed to the LBHB in the active site of citrate synthase by comparing the binding affinities between the carboxyl analog and the primary amine analog of the coenzyme A (Usher et al., 1994). A putative LBHB between Asp56 and a phosphate was also found in the crystal structures of phosphate binding protein (PBP) complexes determined at resolutions of 0.98 and $1.05 \AA$. The $\mathrm{D} 56 \mathrm{~N}$ mutation that converts the LBHB into an ordinary hydrogen bond resulted in no profound effect on the binding affinity of PBP for a phosphate (Wang et al., 1997). These results are consistent with the idea that the additional binding energy and contribution to catalysis provided by an LBHB are not significant compared to those by the ordinary hydrogen bond as observed in the $\mathrm{KSI}$ mutants, Y30F/Y55F/D38N and Y30F/Y55F/Y115F/D38N.

The proton transfer by $\mathrm{KSI}$ from the $\mathrm{C} 4$ proton of 5-AND with $\mathrm{p} K_{\mathrm{a}}$ of 12.7 to the carboxylate oxygen of Asp38 with $\mathrm{p} K_{\mathrm{a}}$ of 4.5 is energetically unfavorable in solution; calculation indicates that it requires $11 \mathrm{kcal} / \mathrm{mol}$ of energy (Ha et al., 2000). This high energetic barrier to proton abstraction during the reaction of KSI should be overcome by a very strong interaction; this requirement seems to contradict our observation that LBHB provides only moderate stabilization of the intermediate. However, the energetic barrier in the reaction of $\mathrm{KSI}$ might be much smaller than $11 \mathrm{kcal} / \mathrm{mol}$, because the $\mathrm{p} K_{\mathrm{a}}$ difference between the carboxylate of Asp38 and the C4-proton of the steroid substrate can be significantly reduced in the active site of $\mathrm{KSI}$ by $\mathrm{pK}$ perturbation ( $\mathrm{Ha}$ et al., 2000); crystal structure of wild-type $P$. putida KSI complexed with deoxycholate, a competitive inhibitor of $\mathrm{KSI}$, revealed that the isopentanoate moiety on the steroid $\mathrm{D}$ ring was involved in hydrogen bonds with the three catalytic residues, Tyr14, Asp38 and Asp99. Analysis of the hydrogen bonding geometry indicated that Asp38, for which estimated $\mathrm{p} K_{\mathrm{a}}=4.5$ (Zhao et al., 1995), might be in the protonated state at $\mathrm{pH} 6.7$ for the formation of the energetically favorable hydrogen bonds between the inhibitor and catalytic residues ( $\mathrm{Ha}$ et al., 2000). Assuming that the stabilization energy of the intermediate can be significantly reduced due to the $\mathrm{p} K_{\mathrm{a}}$ perturbation of a catalytic base (Asp38), explanation of the diffusion controlled reaction rate of $\mathrm{KSI}$ may not require an unusually strong interaction provided by LBHB. The nature of hydrogen bonding in the stabilization of the intermediate was investigated previously in the reaction of KSI by analyzing the binding of substituted phenols to the D38N KSI (Petrounia and Pollack, 1998). The negative charge in the D38N KSI-phenol complex was localized almost exclusively on the bound ligand, with very little charge transfer to the hydrogen bonding groups of the enzyme (Petrounia and Pollack, 1998); this localization is characteristic of ordinary hydrogen bonds. These results indicate that the ordinary hydrogen bond rather than an LBHB would be sufficient to account for the stabilization of the dienolate intermediate during the reaction of $\mathrm{KSI}$.

In conclusion, we have shown that the conversion of LBHB to an ordinary hydrogen bond resulted in only marginal decrease of the catalytic activity and the binding affinity for the intermediate analogue in $P$. putida KSI. Our results indicate that LBHB seems to provide only moderate stabilization of the intermediate in the reaction catalyzed by $P$. putida KSI. Consequently, the enormous rate enhancement achieved by $\mathrm{KSI}$ is not attributed solely to the presence of LBHB in the active site. Rather, the enhancement may be due to the sum of many different contributions including hydrogen bonds, electrostatic and hydrophobic interactions, and positioning of the substrate on the enzyme.

Note: Supplementary information is available on the Molecules and Cells website (www.molcells.org).

\section{ACKNOWLEDGMENTS}

This research was supported by a grant from the National R \& D Program for Cancer Control, Ministry for Health and Welfare, Republic of Korea (1320240). This study was also supported by the National Research Foundation of Korea (NRF) grant funded by the Korea government (MSIP) (NRF-2014R1A2A2A01002931). We thank the staff at $5 \mathrm{C}$ beamline, Pohang Accelerator Laboratory, Korea, for help with the data collection, and Jae-Hee Jeong and Dr. Yeon-Gil Kim for technical assistance and helpful discussion.

\section{REFERENCES}

Afonine, P.V., Grosse-Kunstleve, R.W., Echols, N., Headd, J.J., Moriarty, N.W., Mustyakimov, M., Terwilliger, T.C., Urzhumtsev, A., Zwart, P.H., and Adams, P.D. (2012). Towards automated crystallographic structure refinement with phenix.refine. Acta Crystallogr. D Biol. Crystallogr. 68, 352-367.

Ash, E.L., Sudmeier, J.L., De Fabo, E.C., and Bachovchin, W.W. (1997). A low-barrier hydrogen bond in the catalytic triad of serine proteases? Theory versus experiment. Science 278, 
1128-1132.

Cha, H.J., Jang, D.S., Kim, Y.G., Hong, B.H., Woo, J.S., Kim, K.T. and Choi, K.Y. (2013). Rescue of deleterious mutations by the compensatory Y30F mutation in ketosteroid isomerase. Mol. Cells 36, 39-46.

Cha, H.J., Jeong, J.H., Rojviriya, C., and Kim, Y.G. (2014). Structure of Putrescine Aminotransferase from Escherichia coli Provides Insights into the Substrate Specificity among Class III Aminotransferases. PLoS One 9, e113212.

Cho, H.S., Choi, G., Choi, K.Y., and Oh, B.H. (1998). Crysta structure and enzyme mechanism of Delta 5-3-ketosteroid isomerase from Pseudomonas testosteroni. Biochemistry 37, 8325-8330.

Cho, H.S., Ha, N.C., Choi, G., Kim, H.J., Lee, D., Oh, K.S., Kim, K.S., Lee, W., Choi, K.Y., and Oh, B.H. (1999). Crystal structure of delta(5)-3-ketosteroid isomerase from Pseudomonas testosteroni in complex with equilenin settles the correct hydrogen bonding scheme for transition state stabilization. J. Biol. Chem. 274, 32863-32868.

Cleland, W.W., and Kreevoy, M.M. (1994). Low-barrier hydrogen bonds and enzymic catalysis. Science 264, 1887-1890.

Cleland, W.W., Frey, P.A., and Gerlt, J.A. (1998). The low barrier hydrogen bond in enzymatic catalysis. J. Biol. Chem. 273 25529-25532.

Emsley, P., Lohkamp, B., Scott, W.G., and Cowtan, K. (2010). Features and development of Coot. Acta Crystallogr. D Biol. Crystallogr. 66, 486-501.

Frey, P.A., Whitt, S.A., and Tobin, J.B. (1994). A low-barrier hydrogen bond in the catalytic triad of serine proteases. Science 264, 1927-1930.

Fuhrmann, C.N., Daugherty, M.D., and Agard, D.A. (2006) Subangstrom crystallography reveals that short ionic hydrogen bonds, and not a His-Asp low-barrier hydrogen bond, stabilize the transition state in serine protease catalysis. J. Am. Chem Soc. 128, 9086-9102.

Gerlt, J.A., Kozarich, J.W., Kenyon, G.L., and Gassman, P.G. (1991). Electrophilic catalysis can explain the unexpected acidity of carbon acids in enzyme-catalyzed reactions. J. Am. Chem. Soc. 113, 9667-9669.

Gerlt, J.A., and Gassman, P.G. (1993). Understanding the rates of certain enzyme-catalyzed reactions: proton abstraction from carbon acids, acyl-transfer reactions, and displacement reactions of phosphodiesters. Biochemistry 32, 11943-11952.

Ha, N.C., Kim, M.S., Lee, W., Choi, K.Y., and Oh, B.H. (2000). Detection of large pKa perturbations of an inhibitor and a catalytic group at an enzyme active site, a mechanistic basis for catalytic power of many enzymes. J. Biol. Chem. 275, 4110041106

Ha, N.C., Choi, G., Choi, K.Y., and Oh, B.H. (2001). Structure and enzymology of Delta5-3-ketosteroid isomerase. Curr. Opin Struct. Biol. 11, 674-678.

Jang, D.S., Cha, H.J., Cha, S.S., Hong, B.H., Ha, N.C., Lee, J.Y., Oh, B.H., Lee, H.S., and Choi, K.Y. (2004). Structural doublemutant cycle analysis of a hydrogen bond network in ketosteroid isomerase from Pseudomonas putida biotype B. Biochem. J. 382, 967-973

Jang, D.S., Lee, H.J., Lee, B., Hong, B.H., Cha, H.J., Yoon, J., Lim, K., Yoon, Y.J., Kim, J., Ree, M., et al. (2006). Detection of an intermediate during the unfolding process of the dimeric ketosteroid isomerase. FEBS Lett. 580, 4166-4171.

Kim, S.W., and Choi, K.Y. (1995). Identification of active site residues by site-directed mutagenesis of delta 5-3-ketosteroid isomerase from Pseudomonas putida biotype B. J. Bacteriol. 177, 2602-2605

Kim, S.W., Cha, S.S., Cho, H.S., Kim, J.S., Ha, N.C., Cho, M.J., Joo, S., Kim, K.K., Choi, K.Y., and Oh, B.H. (1997a). High-resolution crystal structures of delta5-3-ketosteroid isomerase with and without a reaction intermediate analogue. Biochemistry 36, 14030-14036.

Kim, S.W., Joo, S., Choi, G., Cho, H.S., Oh, B.H., and Choi, K.Y (1997b). Mutational analysis of the three cysteines and active-site aspartic acid 103 of ketosteroid isomerase from Pseudomonas putida biotype B. J. Bacteriol. 179, 7742-7747.

Kim, D.H., Nam, G.H., Jang, D.S., Choi, G., Joo, S., Kim, J.S., Oh, B.H., and Choi, K.Y. (1999). Roles of active site aromatic residues in catalysis by ketosteroid isomerase from
Pseudomonas putida biotype B. Biochemistry 38, 13810-13819. Kim, D.H., Jang, D.S., Nam, G.H., Choi, G., Kim, J.S., Ha, N.C., Kim, M.S., Oh, B.H., and Choi, K.Y. (2000). Contribution of the hydrogen-bond network involving a tyrosine triad in the active site to the structure and function of a highly proficient ketosteroid isomerase from Pseudomonas putida biotype B. Biochemistry 39, 4581-4589.

Kuliopulos, A., Mildvan, A.S., Shortle, D., and Talalay, P. (1989). Kinetic and ultraviolet spectroscopic studies of active-site mutants of delta 5-3-ketosteroid isomerase. Biochemistry 28, 149-159.

Otwinowski, Z., and Minor, W. (1997). Processing of X-ray diffraction data collected in oscillation mode. Meth. Enzymol. 276, 307-326

Perrin, C.L. (2010). Are short, low-barrier hydrogen bonds unusually strong? Acc. Chem. Res. 43, 1550-1557.

Petrounia, I.P., and Pollack, R.M. (1998). Substituent effects on the binding of phenols to the D38N mutant of 3-oxo-delta5-steroid isomerase. A probe for the nature of hydrogen bonding to the intermediate. Biochemistry 37, 700-705

Plateau, P., and Gueron, M. (1982). Exchangeable proton NMR without base-line distorsion, using new strong-pulse sequences. J. Am. Chem. Soc. 104, 7310-7311.

Pollack, R.M. (2004). Enzymatic mechanisms for catalysis of enolization: ketosteroid isomerase. Bioorg. Chem. 32, 341-353.

Pollack, R.M., Thornburg, L.D., Wu, Z.R., and Summers, M.F. (1999). Mechanistic insights from the three-dimensional structure of 3-oxo-Delta(5)-steroid isomerase. Arch. Biochem. Biophys. 370, 9-15.

Schutz, C.N., and Warshel, A. (2004). The low barrier hydrogen bond (LBHB) proposal revisited: the case of the Asp... His pair in serine proteases. Proteins 55, 711-723.

Shan, S.O., Loh, S., and Herschlag, D. (1996). The energetics of hydrogen bonds in model systems: implications for enzymatic catalysis. Science 272, 97-101.

Stratton, J.R., Pelton, J.G., and Kirsch, J.F. (2001). A novel engineered subtilisin BPN' lacking a low-barrier hydrogen bond in the catalytic triad. Biochemistry 40, 10411-10416.

Usher, K.C., Remington, S.J., Martin, D.P., and Drueckhammer, D.G. (1994). A very short hydrogen bond provides only moderate stabilization of an enzyme-inhibitor complex of citrate synthase. Biochemistry 33, 7753-7759.

Wang, Z., Luecke, H., Yao, N., and Quiocho, F.A. (1997). A low energy short hydrogen bond in very high resolution structures of protein receptor-phosphate complexes. Nat. Struct. Biol. 4, 519-522.

Warshel, A., and Papazyan, A. (1996). Energy considerations show that low-barrier hydrogen bonds do not offer a catalytic advantage over ordinary hydrogen bonds. Proc. Natl. Acad. Sci. USA 93, 13665-13670.

Wu, Z.R., Ebrahimian, S., Zawrotny, M.E., Thornburg, L.D., PerezAlvarado, G.C., Brothers, P., Pollack, R.M., and Summers, M.F. (1997). Solution structure of 3-oxo-delta5-steroid isomerase. Science 276, 415-418

Xue, L.A., Talalay, P., and Mildvan, A.S. (1991). Studies of the catalytic mechanism of an active-site mutant (Y14F) of delta 5-3ketosteroid isomerase by kinetic deuterium isotope effects. Biochemistry 30, 10858-10865.

Zhang, C. (2007). Low-barrier hydrogen bond plays key role in active photosystem II--a new model for photosynthetic water oxidation. Biochim. Biophys. Acta. 1767, 493-499.

Zhao, Q., Li, Y.K., Mildvan, A.S., and Talalay, P. (1995). Ultraviolet spectroscopic evidence for decreased motion of the active site tyrosine residue of delta 5-3-ketosteroid isomerase by steroid binding. Biochemistry 34, 6562-6572.

Zhao, Q., Abeygunawardana, C., Talalay, P., and Mildvan, A.S (1996). NMR evidence for the participation of a low-barrier hydrogen bond in the mechanism of delta 5-3-ketosteroid isomerase. Proc. Natl. Acad. Sci. USA 93, 8220-8224.

Zhao, Q., Abeygunawardana, C., Gittis, A.G., and Mildvan, A.S. (1997). Hydrogen bonding at the active site of delta 5-3ketosteroid isomerase. Biochemistry 36, 14616-14626.

Zhao, L., Liao, H., and Tsai, M.D. (2004). The catalytic role of aspartate in a short strong hydrogen bond of the Asp274-His32 catalytic dyad in phosphatidylinositol-specific phospholipase $C$ can be substituted by a chloride ion. J. Biol. Chem. 279, 3199532000 